\title{
Numerical Study of Single Bubble Growth on and Departure from a Horizontal Superheated Wall by Three-dimensional Lattice Boltzmann Method
}

\author{
Yuan Feng ${ }^{1} \cdot$ Hui-Xiong $\mathrm{Li}^{1}{ }^{1}$ (D) Kai-Kai Guo ${ }^{1} \cdot$ Jian-Fu Zhao $^{2,3} \cdot$ Tai Wang $^{4}$
}

Received: 11 December 2017 / Accepted: 30 April 2018 / Published online: 21 May 2018

(C) Springer Science+Business Media B.V., part of Springer Nature 2018, corrected publication 2018

\begin{abstract}
A three-dimensional hybrid lattice Boltzmann method was used to simulate the progress of a single bubble's growth and departure from a horizontal superheated wall. The evolutionary process of the bubble shapes and also the temperature fields during pool nucleate boiling were obtained and the influence of the gravitational acceleration on the bubble departure diameter (BDD), the bubble release frequency (BRF) and the heat flux on the superheated wall was analyzed. The simulation results obtained by the present three-dimensional numerical studies demonstrate that the BDD is proportional to $g^{-0.301}$, the BRF is proportional to $g^{-0.58}$, and the averaged wall heat flux is proportional to $g^{0.201}$, where $g$ is the gravitational acceleration. These results are in good agreement with the common-used experimental correlations, indicating the rationality of the present numerical model and results.
\end{abstract}

Keywords Three-dimensional lattice Boltzmann method · Pool nucleate boiling · Bubble departure diameter ·

Bubble release frequency

\section{Introduction}

Vapor bubble's growth on and departure from a heated wall are the basic physical processes observed in pool nucleate boiling of liquids. During the pool nucleate boiling, the bubble's behaviors are closely related to the mass and heat transfer between the vapor bubble and the surrounding

This article belongs to the Topical Collection: Approaching the Chinese Space Station - Microgravity Research in China Guest Editors: Jian-Fu Zhao, Shuang-Feng Wang

\footnotetext{
Hui-Xiong Li

huixiong@mail.xjtu.edu.cn
}

1 State Key Laboratory of Multiphase Flow in Power Engineering, Xi' an Jiaotong University, Xi' an 710049, China

2 CAS Key Laboratory of Microgravity, Institute of Mechanics, Chinese Academy of Sciences, Beijing 100190, China

3 School of Engineering Science, University of Chinese Academy of Sciences, Beijing 100049, China

4 School of Energy, Power and Mechanical Engineering, North China Electric Power University, Baoding 071003, China fluids. Therefore, the study on the interaction mechanism between bubble dynamics and boiling heat transfer has great significance for many engineering applications with boiling involved. Early investigations on pool nucleate boiling were mainly conducted by experiments (Cole 1960; Dhir 1991; Dhir et al. 2012; Nikolayev et al. 2015; Wu et al. 2016) among the others). Although these experimental investigations are accurate and reliable, they however have the disadvantages, such as high cost, long time period, and limited data obtained. More importantly, it's hard to investigate the effect of gravitational acceleration on bubbles' dynamics and the related heat transfer process by such experiments. Recently, the rapid development of computer science and technology and numerical simulation technologies provide a new reliable method for the investigation on bubbles' dynamics and related pool nucleate boiling phenomena (Son et al. 2001; Mukherjee and Kandlikar 2007; Li et al. 2015; Zhang et al. 2015). Lattice Boltzmann method (LBM) is one of the most effective numerical method that can be used in such studies.

LBM is a mesoscopic method which can intuitively and easily simulate the interaction between fluid and the surrounding environment. Compared with the traditional numerical method, LBM has more advantages in the 
description of complex flow phenomena such as multicomponent, multiphase system and interface dynamics. Recently, several LB models were developed for multiphase and multi-component flows, such as Rothman and Keller's (1980) color method, Shan and Chen's (1993) potential method and Swift et al.'s (1995) free energy method. These models have not been used in practical problems because they are not applicable to actual multiphase system with large density ratio. Unlike these methods, the improved free energy method proposed by Zheng et al. (2006) can be used to simulate the multiphase flows with large density ratio. This method used a relatively accurate Cahn-Hilliard (C-H) equation to define and track the gas-liquid interface without the artificial disposal. With the advantages of simplicity and stability, this model can accurately track the gas-liquid interface and sufficiently keep the Galilean invariance.

Up till now, many scholars have simulated bubble dynamics in pool during nucleate boiling based on the potential method or its improved method. Hazi and Markus (2009) added a phase change model to the potential method and simulated a bubble's growth and departure from a horizontal superheated wall during pool nucleate boiling and flow boiling with however low velocities, and they concluded that the bubble departure diameter (BDD) and the bubble release frequency (BRF) are proportional to $g^{-0.5}$ and $g^{0.75}$, respectively. Gong and Cheng (2012) proposed a new phase change model based on the entropy balance equation and used this model to simulate the process of pool nucleate boiling. They also concluded that BDD and BRF are proportional to $g^{-0.5}$ and $g^{0.75}$, respectively (Gong and Cheng 2012, 2013). In addition, they studied the effects of wall superheat, contact angle on BDD and BRF. However, they did not give the distribution of temperature fields during nucleate boiling. Rostamzadeh et al. (2016) simulated the pool nucleate boiling with nanofluid. They concluded that BDD is proportional to $g^{-0.51}$ and the BBD is smaller for the nanofluid compared with its base liquid. In sum, these numerical studies concluded that BDD is proportional to $g^{-0.5}$. However, without exception their conclusions were based on two-dimensional simulations.

Some other scholars have numerically studied the pool nucleate boiling based on Zheng's (2006) improved free energy model. Dong et al. (2010) firstly added source terms to the $\mathrm{C}-\mathrm{H}$ equation and energy equation to define the phasechange and latent heat, respectively. This new hybrid model was applied to simulate the bubble growth and departure from a superheated wall, and the effects of gravitational acceleration, wall superheat, surface tension force and fluidsolid interaction parameter on BDD were investigated. In terms of gravitational acceleration's effect, they found that
BDD is proportional to $g^{-0472}$. Using a similar numerical method, Ryu and Ko (2012) simulated the progress of pool nucleate boiling. They found that BDD is proportional to $g^{-0.5}$. In addition, they investigated the influence of nucleation site density $(n)$ and wall superheat on averaged heat flux $(q)$ and they found that averaged heat flux is proportional to $n^{3 / 8}$. It is worth mentioning that the law of $\mathrm{BDD} \propto g^{-0.5}$ was also concluded by Dong et al. (2010) and Ryu (2012). However, their conclusion were also based on two-dimensional simulations. Sun and Li (2013a) simulated the pool nucleate boiling by three-dimensional LBM and they found that BDD is proportional to $g^{-0.346}$ This result is different from other results by two-dimensional simulations.

As mentioned above, though LBM method was adopted by many scholars to study the pool nucleate boiling, there are still some problems that need to be solved. Firstly, a majority of previous studies adopted the potential method as the multiphase flow model, however numerical simulations of pool nucleate boiling based on Zheng's (2006) free energy method are rare. It should be noted that the potential method cannot explicitly describe the evolution of the liquid-vapor interface because the region with nonzero gradient of density difference is regarded as the interface. In addition, the potential method isn't applicable for the simulation of actual multiphase system with large density ratio. Secondly, previous studies on vapor bubble dynamics during nucleate boiling were performed mainly under two-dimensional condition, but the related threedimensional simulations are extremely rare. Undeniable, the two-dimensional simulations are quite different from the real cases. Thirdly, although the influence of gravitational acceleration on BDD and BRF has been studied by plenty of scholars, most of the previous numerical results were obtained based on two-dimensional simulations. Finally, although three-dimensional simulations about pool nucleate boiling has been performed by Sun's (2013a), they only studied the influence of gravitational acceleration on BDD. However, the BRF and averaged wall heat flux are also important parameters, thus further three-dimensional numerical study is needed to be performed to investigate the influence of gravitational acceleration on BRF and averaged wall heat flux.

To solve some of the above-mentioned problems, a three-dimensional numerical study of vapor bubble growth on and departure from a horizontal superheated wall was performed in this paper. The evolutionary processes of the bubble growth and the temperature fields in the pool during nucleate boiling was obtained. Based on the threedimensional numerical results, the influence of gravitational acceleration on bubble departure diameter, bubble release 
frequency and averaged heat flux at superheated wall is also investigated in this study.

\section{Numerical Method \\ Multiphase Flow Model}

Prior to describing the lattice Boltzmann equations, the macroscopic governing equations, including the $\mathrm{C}-\mathrm{H}$ (1) and N-S (2)-(3), to be solved are as follows Zheng et al. (2006)

$\frac{\partial \phi}{\partial t}+\nabla \cdot(\phi \mathbf{u})=\theta_{M} \nabla^{2} \psi_{\phi}$

$\frac{\partial n}{\partial t}+\nabla \cdot(n \mathbf{u})=0$

$\frac{\partial(n \mathbf{u})}{\partial t}+\nabla \cdot(n \mathbf{u u})=-\nabla \cdot \mathbf{P}+\mu \nabla^{2} \mathbf{u}+\mathbf{F}_{b}$

where $\psi_{\phi}$ is the chemical potential, which describes the change in Helmholtz free energy by a small local change in composition. $\theta_{M}$ is the mobility, which indicates the intensity of diffusion. $\mathbf{P}$ is the pressure tensor. $\mathbf{F}_{\mathbf{b}}$ is the body force and $\mathbf{F}_{\mathbf{b}}=\Delta \rho g$, where $\Delta \rho$ is a constant and presents the density difference between two phases and $g$ is the gravitational acceleration. Also, $n$ is the total density and $\phi$ is an order parameter. They are defined as

$n=\frac{\rho_{C}+\rho_{D}}{2}, \quad \phi=\frac{\rho_{C}+\rho_{D}}{2}$

here, if $\rho_{C}$ (or $\rho_{D}$ ) is a liquid density, $\rho_{D}$ (or $\rho_{C}$ ) becomes a vapor density. $\phi$ becomes positive in the region where $\rho_{C}>$ $\rho_{D}$ and negative elsewhere. Thus, it represents two-phase distribution as defined by Swift et al. (1995).

The three-dimensional LB implementation of $\mathrm{C}-\mathrm{H}$ equation follows as

$$
\begin{aligned}
s_{i}\left(\mathbf{x}+\mathbf{e}_{i} \delta t, t+\delta t\right)-s_{i}(\mathbf{x}, t)= & (1-\beta)\left[s_{i}\left(\mathbf{x}+\mathbf{e}_{i} \delta t, t\right)\right. \\
& \left.-s_{i}(\mathbf{x}, t)\right]-\frac{\beta}{1-0.5 \beta} \\
& \times\left[s_{i}(\mathbf{x}, t)-s_{i}^{e q}(\mathbf{x}, t)\right]
\end{aligned}
$$

where $s_{i}$ is the distribution function of order parameter, $\mathbf{e}_{i}$ is the lattice velocity, $i$ is the direction of lattice velocity, and $\beta$ is a constant coefficient which allows to control the diffusion rate between neighboring sites. $\phi$ is calculated by

$\phi=\sum_{i} g_{i}(\mathbf{x}, t)$
The local equilibrium distribution function $s_{i}^{e q}$ in Eq. 6 can be calculated by Sun and Li (2013a)

$$
\begin{aligned}
s_{i}^{e q} & =\eta_{i}+\varsigma_{i} \phi+\vartheta_{i} \phi \mathbf{e}_{i} \cdot \mathbf{u}(i=0, \ldots, 6) \\
\eta_{0} & =-3 \Gamma \psi_{\phi}, \eta_{1}=\frac{1}{2} \Gamma \psi_{\phi}(i=1, \ldots, 6) \\
\Gamma & =\frac{\theta_{M}}{0.5 \beta(\beta+1)} \\
\varsigma_{0} & =1, \varsigma_{1}=0(i=1, \ldots, 6) \\
\vartheta_{i} & =\frac{1}{2 \beta}(i=0, \ldots, 6)
\end{aligned}
$$

The D3Q7 model is used to solve (5-7) and the corresponding lattice velocities are defined as

$\left(\mathbf{e}_{0}, \mathbf{e}_{1}, \mathbf{e}_{2}, \mathbf{e}_{3}, \mathbf{e}_{4}, \mathbf{e}_{5}, \mathbf{e}_{6}\right)=\left[\begin{array}{ccccccc}0 & 1 & -1 & 0 & 0 & 0 & 0 \\ 0 & 0 & 0 & 1 & -1 & 0 & 0 \\ 0 & 0 & 0 & 0 & 0 & 1 & -1\end{array}\right]$

The chemical potential $\psi_{\phi}$ in Eq. 1 can be obtained by Zheng et al. (2006)

$$
\begin{aligned}
\psi_{\phi} & =4 \gamma\left[\phi^{3}-(0.5 \times \Delta \rho)^{2} \phi\right]-k \nabla^{2} \phi \\
\gamma & =\frac{3 \sigma}{4 W(0.5 \times \Delta \rho)^{4}}, \quad k=\frac{1}{8} \gamma W^{2}(\Delta \rho)^{2}
\end{aligned}
$$

where $\sigma$ is surface tension coefficient and $W$ is thickness of interface layer.

The three-dimension LB implementation of N-S equations can be described as

$r_{i}\left(\mathbf{x}+\mathbf{e}_{i} \delta t, t+\delta t\right)=r_{i}(\mathbf{x}, t)+\Omega_{i}^{r}$

$$
\begin{aligned}
\Omega_{i}^{r}= & \frac{r_{i}^{e q}(\mathbf{x}, t)-r_{i}(\mathbf{x}, t)}{\tau_{n}}+\left(1-\frac{1}{2 \tau_{n}}\right) \\
& \times \frac{w_{i}}{\frac{1}{2} c^{2}}\left[\left(\mathbf{e}_{i}-\mathbf{u}\right)+\frac{\left(\mathbf{e}_{i}-\mathbf{u}\right)}{\frac{1}{3} c^{2}} \mathbf{e}_{i}\right]\left(\psi_{\phi} \nabla \phi+\mathbf{F}_{\mathbf{b}}\right) \Delta t
\end{aligned}
$$

where $r_{i}$ is the distribution function of density, $w_{i}$ is the weighting factors, $\tau_{n}$ is a single relaxation time related to the kinematic viscosity, and $\tau_{n}=\frac{3 v}{\Delta t c^{2}}+0.5$.

The macroscopic quantities such as density and velocity are obtained as follows:

$n=\sum_{i} r_{i}(\mathbf{x}, t)$ 
$\mathbf{u}=\frac{1}{n}\left[\sum_{i} \mathbf{e}_{i} r_{i}(\mathbf{x}, t)+\frac{1}{2}\left(\psi_{\phi} \nabla \phi+\mathbf{F}_{\mathbf{e x}}\right)\right]$

The local equilibrium distribution function $r_{i}^{e q}$ in Eq. 10 is calculated by Sun and Li (2013a)

$$
\begin{aligned}
r_{i}^{e q}= & w_{i} \xi_{i}+w_{i} n\left[\frac{3 \mathbf{e}_{i} \cdot \mathbf{u}}{c^{2}}+\frac{4.5\left(\mathbf{e}_{i} \cdot \mathbf{u}\right)^{2}}{c^{4}}-\frac{1.5(\mathbf{u})^{2}}{c^{2}}\right] \\
& \times(i=0, \ldots, 18)
\end{aligned}
$$

$\xi_{0}=3 n-6\left(\phi \psi_{\phi}+\frac{n}{3}\right)$

$\xi_{1}=3 \phi \psi_{\phi}+n \quad(i=1, \ldots, 18)$

The D3Q19 model is used to solve (10) and (13), the corresponding weighting factors are defined as $w_{0}=1 / 3$, $w_{i}=1 / 18$ (for $i=1 \sim 6$ ) and $w_{i}=1 / 36$ (for $i=7^{\sim} 18$ ) and the lattice velocities are defined as

$$
\begin{aligned}
& \left(\mathbf{e}_{0}, \mathbf{e}_{1}, \mathbf{e}_{2}, \mathbf{e}_{3}, \mathbf{e}_{4}, \mathbf{e}_{5}, \mathbf{e}_{6}, \mathbf{e}_{7}, \mathbf{e}_{8}, \mathbf{e}_{9}, \mathbf{e}_{10}, \mathbf{e}_{11}, \mathbf{e}_{12}, \mathbf{e}_{13}, \mathbf{e}_{14}, \mathbf{e}_{15}, \mathbf{e}_{16}, \mathbf{e}_{17}, \mathbf{e}_{18}\right) \\
& =\left[\begin{array}{ccccccccccccccccccc}
0 & 1 & -1 & 0 & 0 & 0 & 0 & 1 & -1 & 1 & -1 & 1 & -1 & 1 & -1 & 0 & 0 & 0 & 0 \\
0 & 0 & 0 & 1 & -1 & 0 & 0 & 1 & -1 & -1 & 1 & 0 & 0 & 0 & 0 & 1 & -1 & 1 & -1 \\
0 & 0 & 0 & 0 & 0 & 1 & -1 & 0 & 0 & 0 & 0 & 1 & -1 & -1 & 1 & 1 & -1 & -1 & 1
\end{array}\right]
\end{aligned}
$$

\section{Phase Change Model}

Before considering the phase change model, it's necessary to solve energy equation to obtain the temperature distribution around the vapor bubble. The macroscopic energy equation is described by Eq. 15 in the absence of phase change model.

$\frac{\partial T}{\partial t}+\nabla \cdot(T \mathbf{u})=\alpha \nabla^{2} T$

where $T$ is the temperature and $\alpha$ is the thermal diffusivity coefficient.

According to Inamuro's (2002) thermal lattice Boltzmann model, the macroscopic energy equation can be converted into the lattice Boltzmann equation as following

$h_{i}\left(\mathbf{x}+\mathbf{e}_{i} \delta t, t+\delta t\right)-h_{i}(\mathbf{x}, t)=-\frac{1}{\tau_{T}}\left[h_{i}(\mathbf{x}, t)-h_{i}^{e q}(\mathbf{x}, t)\right]$

where $\tau_{T}$ is a single relaxation time related to the thermal diffusivity coefficient $\alpha$, and $\tau_{T}=\frac{3 \alpha}{\Delta t c^{2}}+0.5$. The temperature $T$ is obtained as follows:

$T=\sum_{i} h_{i}$

A local equilibrium distribution function in Eq. 16 is expressed as Eq. 18 and D3Q19 model is used to solve (16) and (18).

$h_{i}^{e q}=w_{i} T\left(1+\frac{\mathbf{e}_{i} \cdot \mathbf{u}}{c_{s}^{2}}\right)$

According to Dong's (2010) study, the phase change can be identified by the change of phase order parameter. Thus, a phase-change source term related to phase order parameter is added to the Cahn-Hilliard equation and the corresponding interface capturing (5) can be rewritten as Eq. 19

$$
\begin{aligned}
s_{i}\left(\mathbf{x}+\mathbf{e}_{i} \delta t, t+\delta t\right)= & (1-\beta) s_{i}\left(\mathbf{x}+\mathbf{e}_{i} \delta t, t\right)+\beta s_{i}(\mathbf{x}, t) \\
& -\frac{\beta}{1-0.5 \beta}\left[s_{i}(\mathbf{x}, t)-s_{i}^{e q}(\mathbf{x}, t)\right]+w_{i} \dot{\phi}
\end{aligned}
$$

Based on D3Q7 model, the weighting factors are defined as $w_{0}=1 / 4$ and $w_{i}=1 / 8$ (for $i=1 \sim 6$ ). $\dot{\phi}$ is the phase-change source term which can be calculated by Eq. 20 (Dong et al. 2010).

$\dot{\phi}=-\frac{\rho_{L}^{2}}{\rho_{G}} \frac{J a}{P e}\left(\frac{\partial^{2} T}{\partial x^{2}}\right)$

where $\mathrm{Ja}$ and $\mathrm{Pe}$ are important dimensionless parameters, defined as follows

$J a=C_{p L} \frac{\Delta T}{h_{f g}}, \quad P e=\frac{\rho_{L} u_{c} l_{c} C_{p L}}{\kappa_{L}}$

where $\Delta T$ is the wall superheat, $\kappa_{L}$ is thermal conductivity of liquid, $h_{f g}$ is the latent heat of vaporization and $C_{p L}$ is the specific heat capacity. $l_{c}$ and $u_{c}$ are characteristic length and characteristic velocity, respectively, which are defined as follows Ryu and Ko (2012).

$l_{c}=\sqrt{\frac{\sigma}{g_{e} \Delta \rho}}, \quad u_{c}=\sqrt{g_{e} l_{c}}$

where $g_{e}$ is the earth gravitational acceleration.

What's more, the latent heat term should be added to the energy equations when phase change model is present. Thus Eqs. 15 and 16 should be rewritten as follows

$\frac{\partial T}{\partial t}+\nabla \cdot(T \mathbf{u})=\alpha \nabla^{2} T+\frac{\rho_{G}}{\rho_{L}^{2}} \frac{\dot{\phi}}{J a}$ 


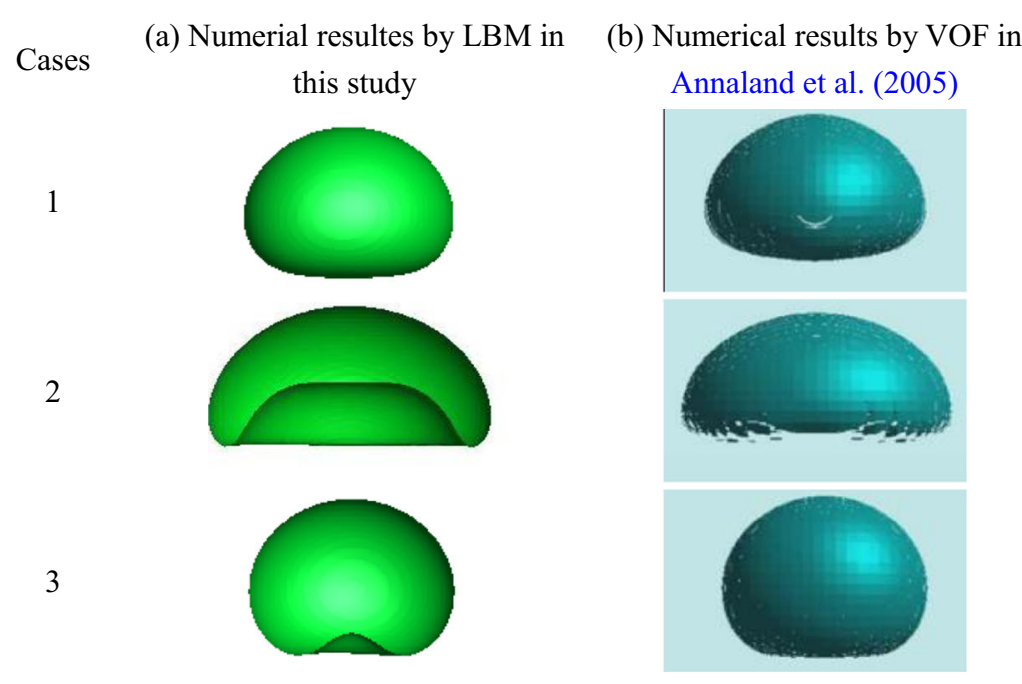

(c) Numerical results by VOSET

in Wang (2016)

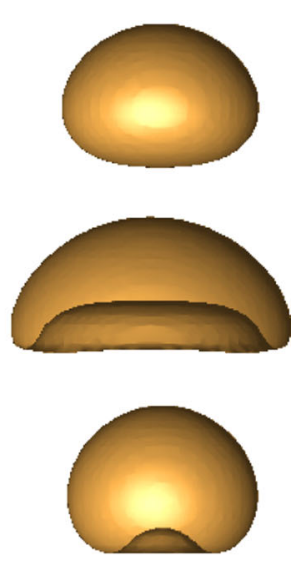

Fig. 1 Bubble shapes simulated by (a) LBM (b) VOF (c) VOSET in different cases

$$
\begin{aligned}
h_{i}\left(\mathbf{x}+\mathbf{e}_{i} \delta t, t+\delta t\right)-h_{i}(\mathbf{x}, t)= & -\frac{1}{\tau_{T}}\left[h_{i}(\mathbf{x}, t)-h_{i}^{e q}(\mathbf{x}, t)\right] \\
& +w_{i} \frac{\rho_{G}}{\rho_{L}^{2}} \frac{\dot{\phi}}{J a}
\end{aligned}
$$

The shapes of a rising vapor bubble after departure are dependent on the Eötvös number $(E o)$ and Morton number $(\mathrm{Mo})$.

$$
E o=\frac{g \Delta \rho l_{c}^{2}}{\sigma}, \quad M o=\frac{g \Delta \rho \mu_{L}^{4}}{\rho_{L}^{2} \sigma^{3}}
$$

\section{Numerical Model Validation}

\section{Validation of Multiphase Flow Model}

The buoyancy-driven motion of a bubble through a viscous liquid was simulated by the three-dimensional LBM to verify the multiphase flow model proposed in the present study, and the numerical results obtained in this study are compared with the numerical results obtained by Annaland et al. (2005) and Wang (2016), as shown in Fig. 1. In these simulations, a bubble with radius $R=16$ is initially located at a cuboid computational domain of $(100 \times 100 \times 200)$ and was released from the position $(50,50,50)$ at the beginning of the computation, and the no-slip boundary condition is adopted in the simulations. The computational conditions for the simulations are taken the same as those by Annaland et al. (2005) and Wang (2016), shown in Table 1.

Figure 1 represents the comparison of the numerical results of bubble shapes obtained by the present numerical method in different cases with those obtained by Annaland et al. (2005) and Wang (2016). As shown in Fig. 1, in case 1,2 and 3 , the numerical results of bubble shapes are ellipsoidal, skirted and dimpled, respectively. In terms of the bubble shapes in each case, the numerical results obtained in this paper are in very good agreement with those of Annaland et al. (2005) and Wang (2016). These numerical results indicate that the multiphase flow model in this paper is pretty reliable.

\section{Validation of Phase Change Model}

In order to validate the phase change model used in this study, a bubble growth in superheated liquid is simulated ( $g=0)$. There is no wall in the computational domain. As an initial condition, a spherical bubble with small radius was positioned at the center of the computational domain. The parameters for the simulations are as follows: the density of liquid $\rho_{L}=1000$, density of vapor $\rho_{G}=1$, surface tension coefficient $\sigma=2$, thickness of phase interface $W=5$ and $J a$ for the simulations are 0.0056 or 0.0112 , respectively. The evolution of bubble radius during the has been collected and depicted in Fig. 2 along with the analytical solutions obtained by Mikic et al. (1970). It can be seen in Fig. 2 that vapor bubble grows continually because the surrounding liquid around the vapor bubble is superheated and provides heat to the bubble continually. In each case, the bubble radius $(R)$ has a tendency of being proportional to the square root of time. This result is consistent with the analytical solutions obtained by Mikic et al. (1970). The comparison

Table 1 Parameters for validation of multiphase flow model

\begin{tabular}{lllll}
\hline Cases & $\rho_{c} / \mathrm{kg} \cdot \mathrm{m}^{-3}$ & $\rho_{d} / \mathrm{kg} \cdot \mathrm{m}^{-3}$ & Eo & Mo \\
\hline 1 & 1000.0 & 1.0 & 10.0 & 0.1 \\
2 & 1000.0 & 1.0 & 100.0 & 1.0 \\
3 & 1000.0 & 1.0 & 100.0 & 1000.0 \\
\hline
\end{tabular}




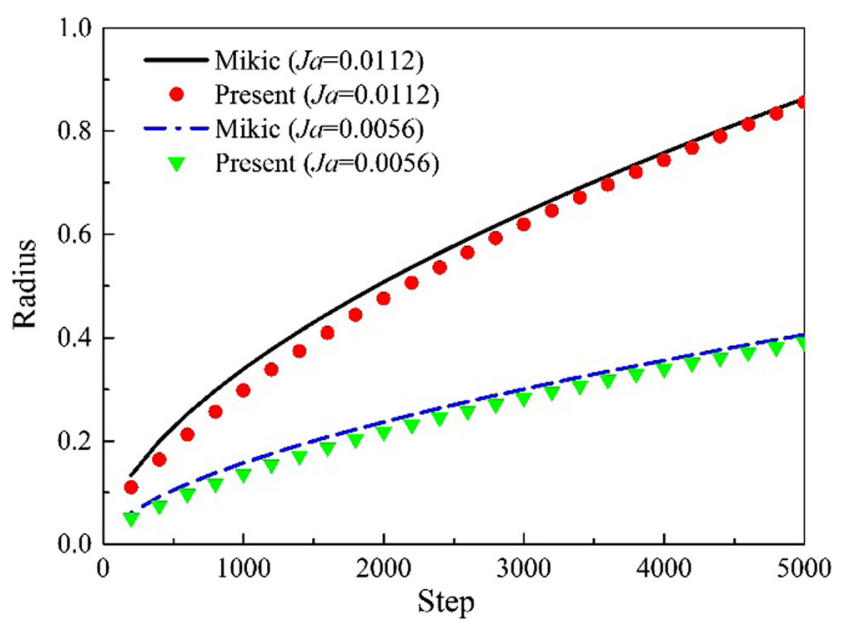

Fig. 2 Comparison in bubble growth between numerical results and analytical solutions by Mikic et al. (1970)

shown in Fig. 2 indicates that the phase change model used in this paper is feasible for the three-dimensional numerical simulation of nucleate boiling.

\section{Numerical Results and Discussions}

In the simulations below, the computational conditions are taken as the same as those in Ryu and Ko (2012). These parameters, including the density, specific heat capacity, thermal conductivity, latent heat of vaporization surface tension coefficient, and contact angle, are shown as follows

$\rho_{L}=1000, \rho_{V}=1, C_{p L}=4.216, \kappa_{L}=0.68$,

$\kappa_{V}=0.025, h_{f g}=2257, \sigma=2, \theta_{W}=53^{\circ}$

What's more, the averaged wall heat flux $(q)$ is an important parameter for this study and it can be calculated by Eq. (26).

$$
\begin{aligned}
q & =\frac{1}{A_{s} f^{-1}} \int_{0}^{f^{-1}} \int_{A_{s}} q_{l o c}(A) d A d t, \\
q_{l o c}(A) & =\left.\kappa(A) \frac{\partial T}{\partial z}\right|_{z=0}
\end{aligned}
$$

where $A_{s}$ is the area of the superheated wall, $q_{l o c}(A)$ is the local heat flux, $\kappa(A)$ is the local thermal conductivity which can be calculated by

$\kappa(A)=\left(\frac{\phi}{\Delta \rho}+0.5\right) \kappa_{L}-\left(\frac{\phi}{\Delta \rho}-0.5\right) \kappa_{G}$

where the order parameter $\phi \in\left[-\frac{\Delta \rho}{2}, \frac{\Delta \rho}{2}\right]$.

The dimensionless time $t^{*}$ is defined as Eq. (27) to represent the same growth stage of vapor bubble during pool nucleate boiling under different conditions.

$t^{*}=\frac{t}{t_{w}+t_{g}}=t f$ where $f$ is the bubble release frequency $t_{w}$ and $t_{g}$ are bubble waiting time and bubble growth period, respectively.

\section{Pool Nucleate Boiling on a Superheated Wall}

(1) Bubble dynamics in normal gravity

Figure 3 represents the evolution of the bubble shapes and temperature fields before bubble departure. In Fig. 3, the gravitational acceleration is set as earth gravitational acceleration $\left(g=g_{e}=9.8 \mathrm{~m} / \mathrm{s}^{2}\right)$, and the wall superheat is set as $5 \mathrm{~K}(\Delta T=5 \mathrm{~K})$. Thus, the dimensionless parameters are $E o=1, M o=9.5 \times 10^{-5}$ and $J a=0.00934$. As shown in Fig. 3a and b, since the bubble volume is small at the beginning of the calculation, almost the entire bubble is immersed in the superheated layers, the bubble grows fast due to heat transfer from the surrounding superheated layers. At this stage, the bubble shape is semi-spherical and the bubble base diameter increases continuously. In Fig. $3 c$ and d, as the bubble volume increases, the temperature gradient at the top position of vapor bubble decreases. At this time, the heat for evaporation is mainly supplied by the bubble base and the bubble growth rate decreases. In Fig. 3e and $\mathrm{f}$, a neck starts to form near the bubble base and the bubble tends to depart away from the superheated wall under the effect of increasing buoyancy forces. As the bubble grew further, the buoyancy forces finally become strong enough to overcome the adhesion force between the vapor bubble and superheated wall, and the bubble departed away from the superheated wall, as shown in Fig. 4. It can be seen from Fig. 4 that the rising vapor bubble is an ellipsoid and the thermal boundary layer is distorted due to the trailing wake of the rising bubble.

(2) Bubble dynamic under microgravity condition

Figure 5 represents the evolution of in bubble shapes and temperature fields during pool nucleate boiling under microgravity condition. Compared with Fig. 3, the gravitational acceleration in Fig. 5 reduces to $g=$ $0.1 g_{e}$, thus the dimensionless parameters are $E o=0.1$, $M o=9.5 \times 10^{-6}$ and $J a=0.00934$. In Fig. 5a-c, similar to Fig. 3a-c, the vapor bubble grows fast due to the heat transfer from the surrounding superheated layers, but the bubble growth rate decreases gradually. In Fig. $5 \mathrm{~d}$ and $\mathrm{e}$, with the increase in bubble volume, buoyancy forces becomes stronger, the vapor bubble tends to depart away from the superheated wall and a neck forms near the bubble base. In Fig. 5f, the bubble starts to rise after its departure. Compared with that in Fig. 4, the bubble rises more slowly in Fig. $5 \mathrm{f}$ because of the reduced gravity.

Figure 6 presents the distribution of phase fields, temperature fields and local heat flux $q$ on superheated wall along the horizontal axis of symmetry. The data 


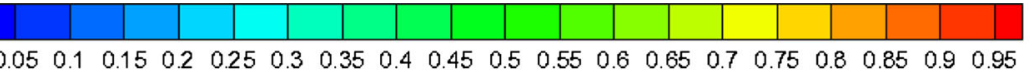

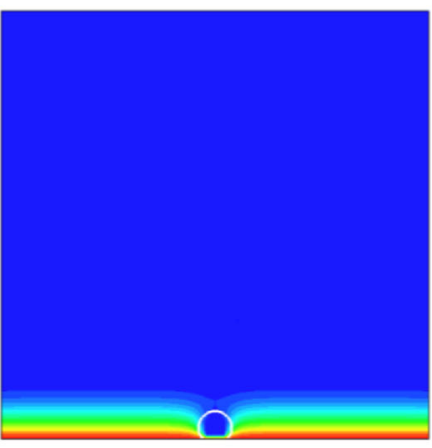

(a) Time steps $=0$

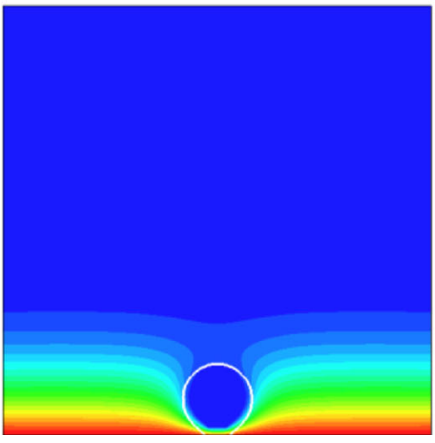

(d) Time steps $=3000$

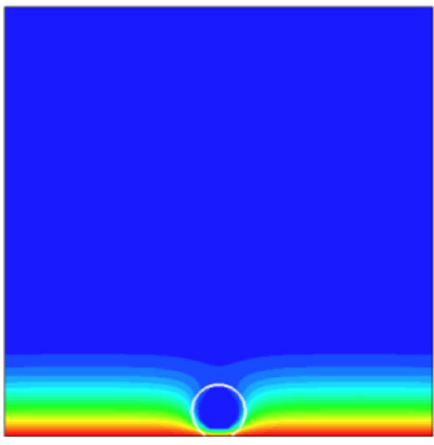

(b) Time steps $=1000$

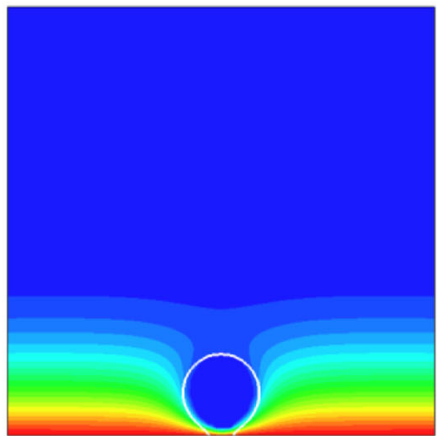

(e) Time steps $=4000$

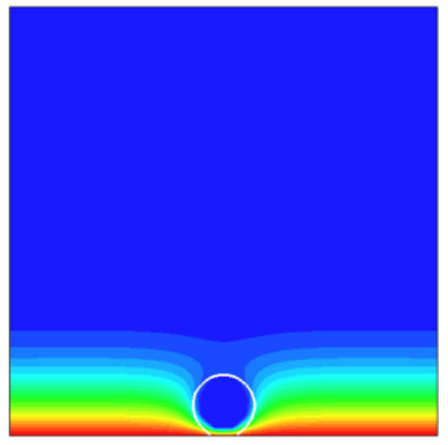

(c) Time steps $=2000$

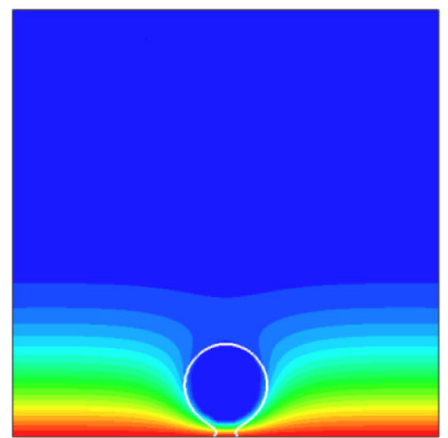

(f) Time steps $=5000$

Fig. 3 Evolution of bubble shapes and temperature profiles with time before bubble departure $(E o=1, J a=0.00934)$

used in Fig. 6 is extracted from Fig. 5c. As shown in Fig. 6, the local heat flux increases gradually outside of the vapor bubble but decreases suddenly inside of the vapor bubble. As a result, the local heat flux reaches a maximum near the contact line. It's because the local heat flux is affected by the temperature difference between wall and the fluid near the wall, as well as the thermal conductivity of fluid near the wall. Outside of the vapor bubble, the local heat flux increases gradually because the temperature difference increases as its position gets closer to the contact line. Inside of the vapor bubble, the local heat flux decreases suddenly because the thermal conductivity of vapor is much smaller than that of liquid.
Fig. 4 Evolution of bubble shapes and temperature profiles with time after bubble departure $(E o=1, J a=0.00934)$

$\begin{array}{lllllllllllllllllllll}0.05 & 0.1 & 0.15 & 0.2 & 0.25 & 0.3 & 0.35 & 0.4 & 0.45 & 0.5 & 0.55 & 0.6 & 0.65 & 0.7 & 0.75 & 0.8 & 0.85 & 0.9 & 0.95\end{array}$

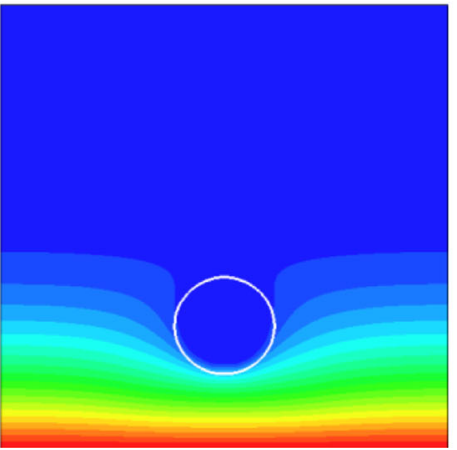

(a) Time steps $=8000$

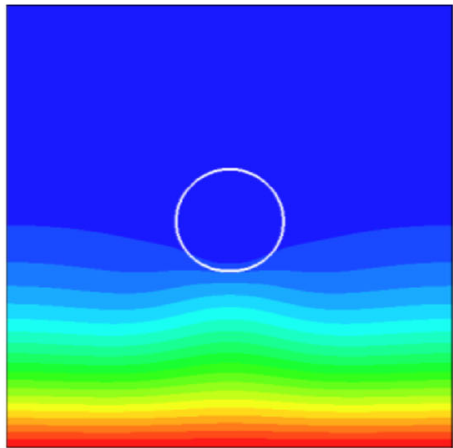

(b) Time steps $=13500$ 
$\begin{array}{lllllllllllllllllllll}0.05 & 0.1 & 0.15 & 0.2 & 0.25 & 0.3 & 0.35 & 0.4 & 0.45 & 0.5 & 0.55 & 0.6 & 0.65 & 0.7 & 0.75 & 0.8 & 0.85 & 0.9 & 0.95\end{array}$

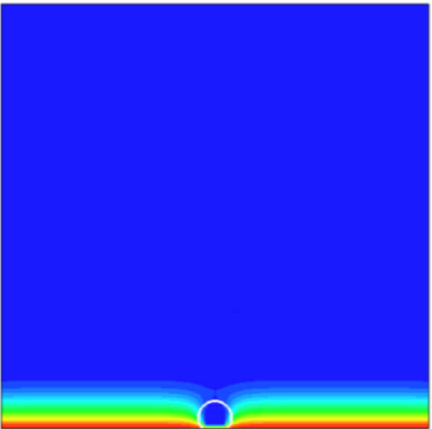

(a) Time steps $=0$

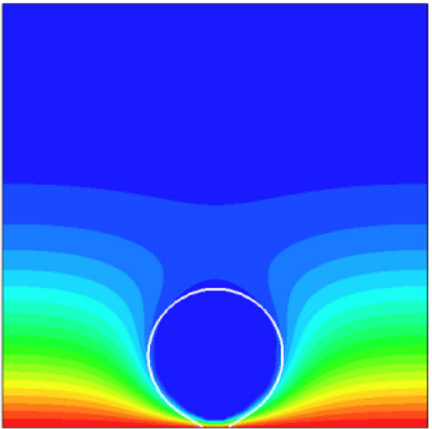

(d) Time steps $=14000$

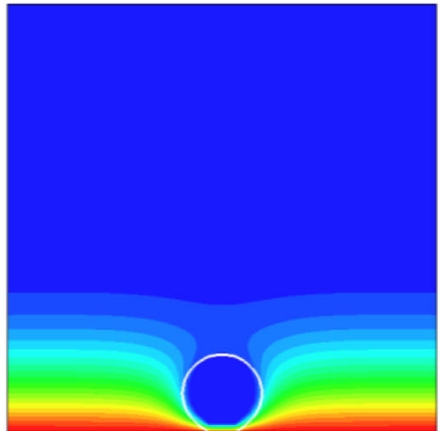

(b) Time steps $=4000$

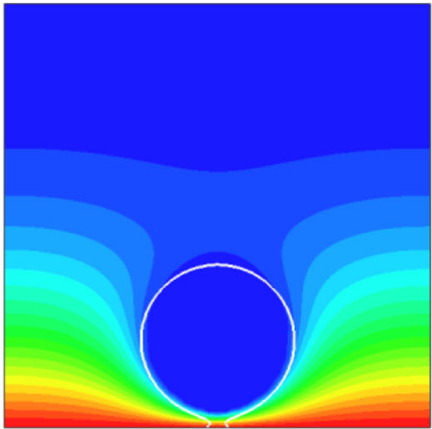

(e) Time steps $=19000$

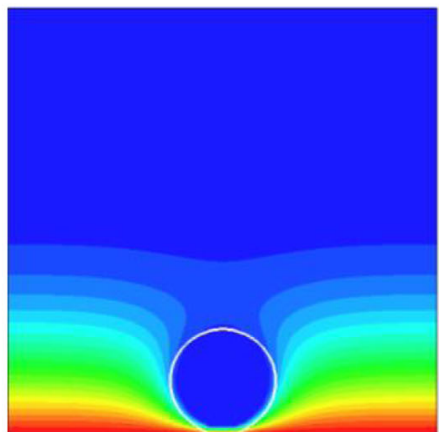

(c) Time steps $=8000$

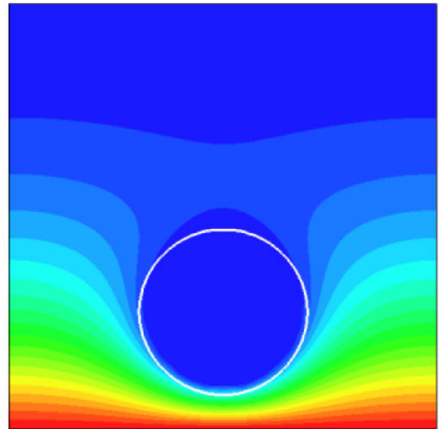

(f) Time steps $=24000$

Fig. 5 Evolution of bubble shapes and temperature profiles with time under microgravity condition $(E o=0.1, J a=0.00934)$

\section{Effect of Gravitational Acceleration on BDD and BRF}

For nucleate boiling, bubble departure diameter (BDD) is an important parameter because $\mathrm{BDD}$ is related to the

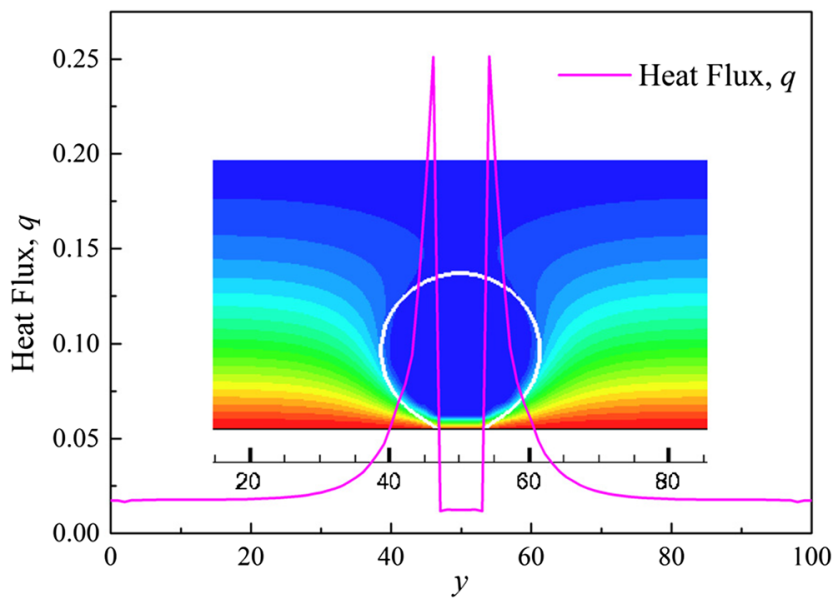

Fig. 6 The distribution of temperature fields, phase fields and local heat flux $q$ on superheated wall along the horizontal axis of symmetry under microgravity condition when $t=8000$ ( $E o=0.1, J a=0.00934$, $x=50, z=0$ ) boiling heat transfer efficiency. Figure 7 represents the three-dimensional numerical results of BDD under different conditions with gravitational accelerations. As shown in Fig. 7, BDD increases with the decrease in gravitational acceleration. By the regression method, it can be found that the BDD is proportional to $g^{-0.301}$.

Table 2 lists the correlations of BDD, which are based on experiments and theoretical studies, proposed by previous scholars. As shown in Table 2, these correlations are quite different in form. In terms of the effect of gravitational acceleration on BDD, it can be found that BDD is proportional to $g^{\varphi}$, where $\varphi$ equals to -0.5 in the correlations of Fritz (1935), Kocamustafaogullari (1983b) and Cole and Rohsenow (1969) and $\varphi$ equals to $-1 / 3$ in the correlation of Gorenflo et al. (1986). On the other hand, by numerical simulations, the relationship between gravitational acceleration and BDD has been studied by Hazi and Markus (2009), Gong and Cheng (2013), Dong et al. (2010) and Ryu and Ko (2012), and all of them concluded that BDD is proportional to $g^{-0.5}$.

Obviously, the numerical results of BDD in Fig. 7 differ from those obtained by Hazi and Markus (2009), Gong and Cheng (2013), Dong et al. (2010), Sun et al. 


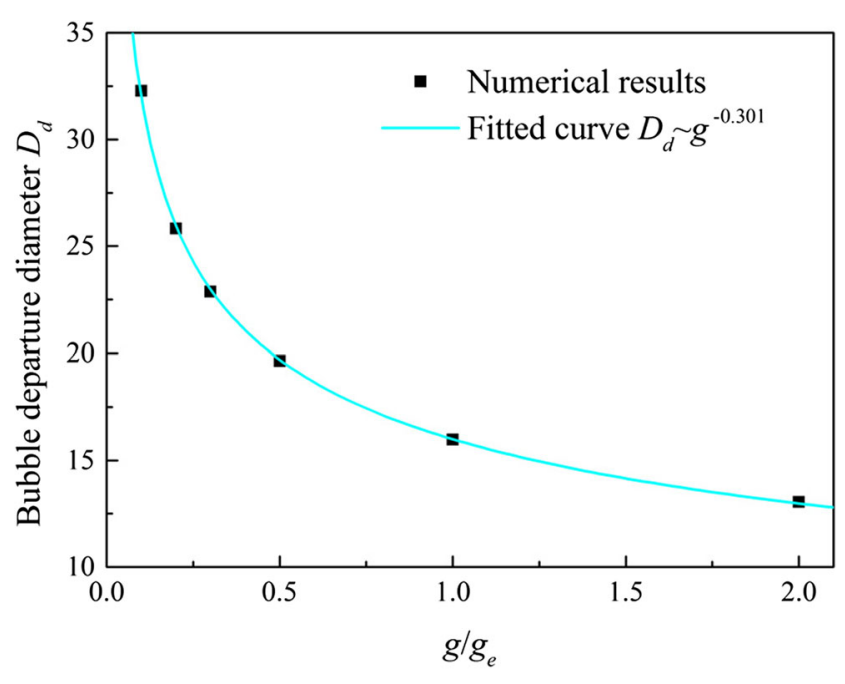

Fig. 7 Effect of gravitational acceleration on bubble departure diameter

(2013b) and Ryu and Ko (2012). It's because their results were based on two-dimensional simulations. However, as can be seen from Fig. 7 and Table 2, the threedimensional numerical results in present study are in good agreement with Sun's (2013a) three-dimensional numerical result and Gorenflo's (1986) correlation. The numerical results indicate the rationality of the numerical model and calculation results and that Gorenflo's (1986) correlation could describe the influence of gravitational acceleration on $D_{d}$ in three-dimensional simulations. It is worth pointing out that Sun et al. have studied the effect of gravitational acceleration on BDD based on both two-dimensional and three-dimensional numerical simulations. They also found that BDD is proportional to $g^{-0.5}$ and $g^{-1 / 3}$ in twodimensional numerical simulations and three-dimensional numerical simulations, respectively.

The correlation of Fritz (1935) was proposed through theoretical analysis. According to hydrostatic principle, Fritz (1935) obtained the correlation by balancing the buoyancy force with surface tension force. However, significant deviations are caused when Fritz's correlation is used to predict BDD in practical boiling, especially at high pressures. The correlations of Cole and Rohsenow (1969), Kocamustafaogullari (1983b) and Gorenflo et al. (1986) were obtained by theoretical analysis and experimental method, and the experimental data at low pressures, high pressures and high heat fluxes were used, respectively. In their studies, the effect of gravitation acceleration on BDD was not included and the gravitation acceleration was just regarded as a constant instead of a variable. In fact, the relationship between BDD and gravitation acceleration in the studies of Cole and Rohsenow (1969) and Kocamustafaogullari (1983b) was derived from the theoretical analysis by Fritz (1935). Whereas the theoretical source of the relationship between BDD and gravitation acceleration in the study of Gorenflo et al. (1986) wasn't given. Besides the correlations in Table 2, Wallis (1969) also obtained a correlation to predict BBD, as shown in Eq. 28.

$D_{d}=2\left[\sigma R_{0} /(\Delta \rho) g\right]^{1 / 3}$

Obviously, the correlation of Wallis (1969) suggested that $D_{d} \propto g^{-1 / 3}$, which was consistent with the correlation of Gorenflo (1986) and the numerical results in this paper. Wallis assumed a bubble was formed at a circular orifice of radius $R_{0}$ facing upward in a stationary fluid and obtained the correlation of BBD by balancing the buoyancy

Table 2 Correlations about bubble departure diameter in published literatures

\begin{tabular}{|c|c|c|c|}
\hline Authors & Correlations & Characteristic & Approach \\
\hline Fritz (1935) & $D_{d}=0.0208 \theta_{W}\left[\frac{\sigma}{g \Delta \rho}\right]^{0.5}$ & $\begin{array}{l}\text { 1) Only contact angle, surface } \\
\text { tension and gravity are taken } \\
\text { into account; } \\
\text { 2) } D_{d} \propto g^{-0.5}\end{array}$ & Theoretical analysis \\
\hline Cole and Rohsenow (1969) & $D_{d}=1.5 \times 10^{-4} \sqrt{\frac{\sigma}{g \Delta \rho}}\left(J a \frac{\rho_{L}}{\rho_{V}}\right)^{\frac{5}{4}}$ & $\begin{array}{l}\text { 1) Influence of } J a \text { is taken into ; } \\
\text { account } \\
\text { 2) } D_{d} \propto g^{-0.5}\end{array}$ & $\begin{array}{l}\text { Theoretical analysis } \\
\text { and data-fitting }\end{array}$ \\
\hline Kocamustafaogullari (1983b) & $D_{d}=2.64 \times 10^{-5} \sqrt{\frac{\sigma}{g \Delta \rho}}\left(\frac{\Delta \rho}{\rho_{V}}\right)^{0.9}$ & $\begin{array}{l}\text { 1) Fluid property parameters } \\
\text { are taken into account; } \\
\text { 2) } D_{d} \propto g^{-0.5}\end{array}$ & $\begin{array}{l}\text { Theoretical analysis } \\
\text { and data-fitting }\end{array}$ \\
\hline Gorenflo et al. (1986) & $D_{d}=C_{1}\left(\frac{J a^{4} \alpha_{L}^{2}}{g}\right)^{\frac{1}{3}}\left[1+\sqrt{1+\frac{2 \pi}{3 J a}}\right]^{\frac{4}{3}}$ & $\begin{array}{l}\text { 1) Fluid property parameters } \\
\text { and } J a \text { are taken into account } \\
\text { 2) } D_{d} \propto g^{-1 / 3}\end{array}$ & $\begin{array}{l}\text { Theoretical analysis } \\
\text { and data-fitting }\end{array}$ \\
\hline
\end{tabular}




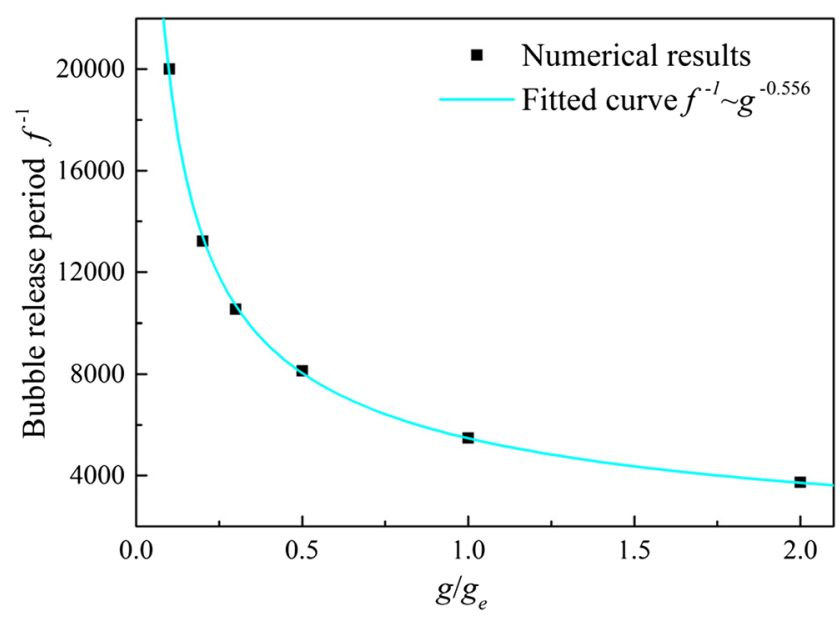

Fig. 8 Effect of gravitational acceleration on the inverse of bubble release frequency

force with surface tension force. In this paper, we found the size of bubble base stayed almost constant when the bubble starts to depart away from the superheated wall and gravitational acceleration had little effect on the size of bubble base. This result is appropriate to the hypothesis of Wallis (1969). Therefore, the numerical results in this paper are most consistent with the correlation of Wallis (1969) and Gorenflo et al. (1986).

It should be noted that there are still some drawbacks in Gorenflo's (1986) correlation. For example, the effects of contact angle and surface tension are not taken into its consideration. Therefore, more efforts must be made to obtain a more accurate correlation for bubble departure diameter and the influences of other parameters, such as wall superheat, contact angle and surface tension should be investigated by three-dimensional numerical simulations.

Bubble release frequency (BRF) is also an important parameter for pool nucleate boiling. BRF is the inverse of the sum of waiting and growth period. However, both of the waiting time $t_{w}$ and growth period $t_{g}$ are difficult to determine quantitatively, thus BRF can't be calculated by $t_{w}$ and $t_{g}$ directly. By the three-dimensional LBM, the numerical results of $f^{-1}$ under different gravitational accelerations are shown in Figure 8. It can be seen that $f^{-1}$ decreases with the increase in gravitational acceleration, thus the detachment of vapor bubble becomes harder under the reduced gravitational acceleration. By the regression method, it can be found that the inverse of BRF is proportional to $g^{-0.556}$.

Table 3 represents the experimental correlations of BRF proposed by previous scholars. It can be seen that predictions of $f$ are often associated with the BDD. In Table 3, all of these correlations are semi-empirical formula obtained by experimental fitting. In general, the accurate relationship between BRF and BDD was ambiguous and these correlations deviated from each other greatly. It's because the physical mechanism between BRF and BDD is not clear and BRF can be affected by much factors, such as temperature fields, cavity-cavity interaction, microlayer evaporation contribution, and bubble merger. However, these factors may be also influenced by gravitational acceleration. Therefore, the relationship between BRF and gravitational acceleration is much complex and difficult. In three-dimensional conditions, if the numerical result of $D_{d} \propto g^{-0.301}$ is substituted into these correlations, the relationship between gravitational acceleration and $f$ could be obtained, as shown in the third column of Table 3. It can be found that the bubble release frequency is proportional to $g^{\lambda}$, where $\lambda$ equals to $-0.301-0.551,-0.651$ and 0.602 in the correlations of Jacob (1949), Zuber (1959), Mcfadden and Grassmann (1962), and Siegel and Keshock (1964), respectively. Obviously, the numerical results of $f^{-1} \propto g^{-0.556}$ in present study is very close to Zuber's correlation, as well as that of Siegel \& Keshock. The correlation of Siegel \&Keshock was obtained based on short-term microgravity experiments. Thus, the agreement mentioned above proves the rationality of numerical model and calculation results once again. The numerical results also indicate that the correlations of Zuber and Siegel \& Keshock can be used to describe the influence of gravitational acceleration on BRF and BDD in threedimensional simulations. It needs to be pointed out that only the effect of gravitational acceleration is investigated in this paper. In order to obtain a more accurate correlation for bubble release frequency by verifying or revising the correlations of Zuber and Siegel \& Keshock, the effects of wall superheat, contact angle and surface tension have to be investigated further.
Table 3 Correlations about bubble release frequency in published literatures

\begin{tabular}{lll}
\hline Authors & Original correlations & $\begin{array}{l}\text { Three-dimensional } \\
\text { results }\left(D_{d} \tilde{g}^{-0.301}\right)\end{array}$ \\
\hline Jacob (1949) & $f D_{d}=$ const & $f^{-1} \propto g^{-0.301}$ \\
Zuber (1959) & $f D_{d}=0.59\left(\frac{\sigma \Delta \rho g}{\rho_{L}^{2}}\right)^{0.25}$ & $f^{-1} \propto g^{-0.551}$ \\
Mcfadden and Grassmann (1962) & $f D_{d}^{0.5}=0.56 \sqrt{g}$ & $f^{-1} \propto g^{-0.651}$ \\
Siegel and Keshock (1964) & $f D_{d}^{2}=$ const & $f^{-1} \propto g^{-0.602}$ \\
\hline
\end{tabular}




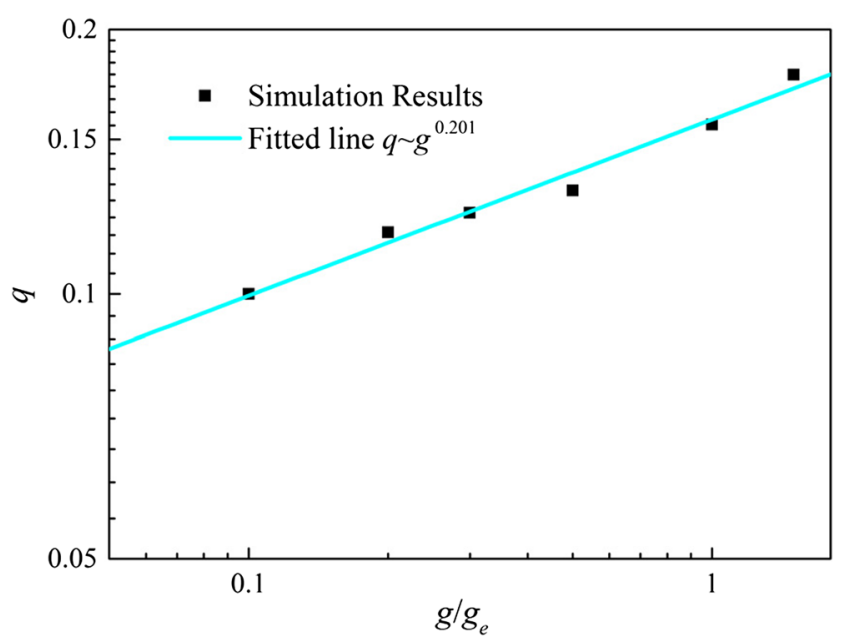

Fig. 9 Influence of gravitational acceleration on averaged wall heat flux

\section{Influence of Gravitational Acceleration on Averaged Wall Heat Flux}

Figure 9 presents the numerical results of averaged heat flux $q$ on superheated wall under different gravitational accelerations. As shown in Fig. 9, averaged heat flux decreases with the decrease in gravitational acceleration. When the gravitational acceleration reduces from $g_{e}$ to $0.1 g_{e}, q$ decreases by about $40 \%$. It's because the BDD increases with the decrease in gravitational acceleration, and the thermal conductivity of vapor is much smaller than that of liquid, while $\kappa_{V} / \kappa_{L} \approx 0.037$. Therefore the boiling heat-transfer capability decreases. By the regression method, it can be found that averaged heat flux $q$ is proportional to $g^{0.201}$.

Table 4 lists the correlations for averaged heat flux in published literatures. As shown in Table 4, these correlations are quite different in form. Kurihara and Myers (1960) studied the effects of liquid superheat and surface roughness on boiling coefficients through a series of experiments in which water, acetone, n-hexane, carbon tetrachloride, and carbon disulfide were boiled on a flat plate. Thus the effects of fluid density, dynamic viscosity coefficient and heat conductivity coefficient were included in the correlation of Kurihara and Myers (1960). The correlation of Tien (1962) was proposed through the established analytical results in axisymmetrical stagnation flow, a relation between the heat-transfer coefficient and the thermal boundary-layer thickness induced by rising bubbles is obtained, and a good agreement with measured results in the low heat-flux region is indicated. In the studies of Kurihara and Myers (1960) and Tien (1962), the effects of gravitational acceleration on averaged heat flux were not included. The correlation of Rohsenow (1952) was proposed by theoretical analysis and experimental method. In his study, the heat flux was expressed as a function of BDD and BRF. The correlations of Fritz (1935) and Jacob (1949) were used to calculate BDD and BRF, thus Rohsenow (1952) suggested that $q$ is proportional to $g^{0.5}$. The correlation of Kocamustafaogullari and Ishii (1983a) was proposed throughy theoretical analysis and expressed as a function of fluid density, specific heat capacity, wall superheat, Prandtl number, BDD and active nucleation site density. After simplification, Kurihara and Myers (1960) suggested that $q$ is proportional to $g^{0.125}$. In sum, Tien (1962) and Kurihara and Myers (1960) suggested that $q$ is independent of gravitational acceleration, Kocamustafaogullari and Ishii (1983a) suggested that $q$ is proportional to $g^{0.125}$ and Rohsenow (1952) suggested that $q$ is proportional to $g^{0.5}$. Therefore, no agreement has been reached in the relationship between $q$ and gravitational acceleration.

As shown in Fig. 9, the three-dimensional numerical results of $q$ are closest to the Kocamustafaogullari's correlation. The difference between numerical results and Kocamustafaogullari's correlation may be caused by two reasons. On the one hand, the effect of BRF on $q$ was not included in Kocamustafaogullari's correlation. On the other hand, the relationship between microlayer evaporation heat flux and gravitation acceleration obtained in this paper was distinct from the formula in Dhir's review. Figure 10 presents the distribution of local heat flux on the superheated wall under different gravitational accelerations at the same $t^{*}$, which could ensure the same growth stage of vapor bubble during pool nucleate boiling. As shown in Fig. 10, the maximum of the local heat flux increases with the increase in gravitational accelerations. However, according to the review by Dhir (2010), the microlayer evaporation heat flux $q_{e} \propto D_{d}^{2} \propto g^{-0.6}$. Obviously, the numerical results obtained in this paper are inconsistent

Table 4 Correlations for averaged wall heat flux in published literatures

\begin{tabular}{llc}
\hline Authors & Correlations & Characteristic \\
\hline Rohsenow (1952) & $\frac{q \sqrt{\frac{\sigma}{g \Delta \rho}}}{\mu_{L} h_{f g}}=C_{s}^{-3}\left[\frac{c_{p l} \Delta T}{h_{f g}}\right] \operatorname{Pr}_{l}^{-n}$ & $q \propto g^{0.5}$ \\
Kurihara and Myers (1960) & $q=36 \kappa_{L} \operatorname{Pr}_{l}^{0.33}\left(\rho_{V} / \mu_{L}\right)^{1 / 3} \Delta T n^{1 / 3}$ & $q \propto g^{0}$ \\
Tien (1962) & $q=61.3 \operatorname{Pr}_{L}^{0.33} \kappa_{L} \Delta T n^{1 / 2}$ & $q \propto g^{0}$ \\
Kocamustafaogullari and Ishii (1983a) & $q=14 \kappa_{L}\left(\frac{\rho_{L} c_{p l}}{\rho_{V} h_{f g}}\right)^{0.5} \operatorname{Pr}_{L}^{-0.39}\left[2.5 \times 10^{-5}\left(\frac{\rho_{L}-\rho_{V}}{\rho_{V}}\right)^{0.9} \theta_{W} \sqrt{\frac{\sigma}{g \Delta \rho}}\right]^{-0.25} \Delta T^{1.5} n^{3 / 8}$ & $q \propto g^{0.125}$ \\
\hline
\end{tabular}




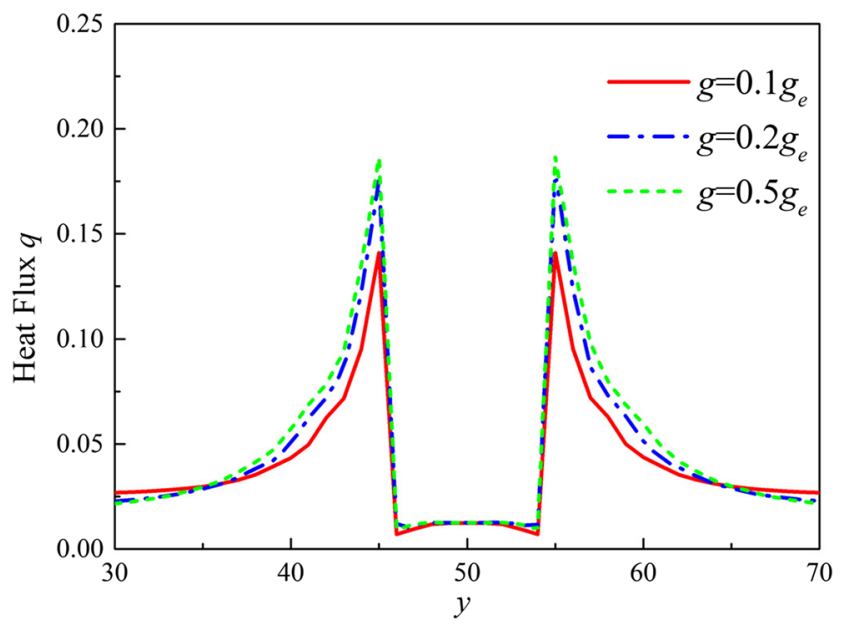

Fig. 10 The distribution of local heat flux on the superheated wall along the horizontal axis of symmetry under different gravitational accelerations at $t^{*}=0.4(\mathrm{Ja}=0.00934, x=50, z=0)$

with the review by Dhir (2010). Thus, the numerical model used in this paper should be improved in future and more detailed investigation on the relationship between $q$ and gravitation acceleration should be investigated. Besides, as shown in Table 4, no agreement has neither been reached in the relationship between $q$ and nucleation site density, wall superheat. Therefore, further investigation by threedimensional numerical method should be performed to obtain a more accurate correlation for heat flux.

\section{Conclusions}

Based on Zheng's (2006) improved free energy model, a three-dimensional hybrid lattice Boltzmann method was used to simulate the dynamic evolution of vapor bubbles growing and departing on a horizontal superheated wall during pool nucleate boiling. The influence of gravitational acceleration on bubble departure diameter, bubble release frequency and averaged heat flux was analyzed on the basis of the three-dimensional numerical results. The conclusions derived from the numerical results can be summarized as follows:

1. With the decrease in gravitational acceleration, the bubble departure diameter increases and the bubble release frequency decreases because the reduced buoyancy forces make it difficult for the bubble depart from the heated surface.

2. The three-dimensional numerical simulations result shows that bubble departure diameter is proportional to $g^{-0.301}$. This result is in good agreement with the correlation proposed by Gorenflo et al. (1986), however, is different from two-dimensional numerical results reported in other literatures. The bubble release frequency obtained in the present simulations is proportional to $g^{-0.556}$. This result is in good agreement with the correlation suggested by Zuber (1959) and Siegel and Keshock (1964).

3. With the decrease in gravitational acceleration, the averaged heat flux on superheated wall decreases, indicating that the heat transfer efficiency of pool nucleate boiling is weakened under reduced gravities. The results of averaged heat flux is proportional to $g^{0.201}$. This result is in closest agreement to the empirical correlation proposed by Kocamustafaogullari and Ishii (1983a).

4. In previously published literatures, the empirical correlations for bubble departure diameter and bubble release frequency are quite different in not only the form of the correlations but also the prediction results of the correlations. Thus, the influences of many parameters, such as wall superheat, surface tension and contact angle of the liquid to the heater surface and so forth on bubble departure diameter and bubble release frequency should be investigated further using three-dimensional numerical methods.

Acknowledgments This work is supported financially by the joint fund between the Chinese Academy of Sciences (CAS) and National Natural Science Foundation of China (NSFC) under the grant of U1738105.

\section{References}

Annaland, M.V., Deen, N.G., Kuipers, J.A.M.: Numerical simulation of gas bubbles behaviour using a three-dimensional volume of fluid method. Chem. Eng. Sci. 60, 2999-3011 (2005)

Cole, R.: A photographic study of pool boiling in the region of critical heat flux. AIChE J. 6, 533-538 (1960)

Cole, R., Rohsenow, W.: Correlation of bubble departure diameters for boiling of saturated liquids. Chem. Eng. Prog. 65, 211-213 (1969)

Dhir, V.K.: Nucleate and transition boiling heat transfer under pool and external flow conditions. Int. J. Heat Fluid Flow 12, 290-314 (1991)

Dhir, V.K.: Boiling heat transfer. Naval Eng. J. 67, 457-466 (2010)

Dhir, V.K., Warrier, G.R., Aktinol, E., Chao, D., Eggers, J., Sheredy, W., Booth, W.: Nucleate pool boiling experiments (NPBX) on the international space station. Microgravity Sci. Technol. 24, 307-325 (2012)

Dong, Z.Q., Li, W.Z., Song, Y.C.: A numerical investigation of bubble growth on and departure from a superheated wall by lattice Boltzmann method. Int. J. Heat Mass Transfer 53, 4908-4916 (2010)

Fritz, W.: Berechnung des maximal Volumens von Dampfblasen. Phys. Z. 379-388, 36 (1935)

Gong, S., Cheng, P.: A lattice Boltzmann method for simulation of liquid-vapor phase-change heat transfer. Int. J. Heat Mass Transfer 55, 4923-4927 (2012)

Gong, S., Cheng, P.: Lattice Boltzmann simulation of periodic bubble nucleation, growth and departure from a heated surface in pool boiling. Int. J. Heat Mass Transfer 64, 122-132 (2013) 
Gorenflo, D., Knabe, V., Bieling, V.: Bubble density on surfaces with nucleate boiling-It's Influence on Heat Transfer and Burnout Heat Fluxes at Elevated Saturation Pressures, vol. 4, Hemisphere (1986)

Hazi, G., Markus, A.: On the bubble departure diameter and release frequency based on numerical simulation results. Int. J. Heat Mass Transfer 52, 1472-1480 (2009)

Inamuro, T., Yoshino, M., Inoue, H., Mizuno, R., Ogino, F.: A lattice Boltzmannmethod for a binary miscible fluid mixture and its application to a heat-transfer problem. J. Comput. Phys. 179, 201-215 (2002)

Jacob, M.: Heat Transfer. Wiley, New York (1949)

Kocamustafaogullari, G., Ishii, M.: Interfacial area and nucleation site density in boiling systems. Int. J. Heat Mass Transfer 26, 1377-1387 (1983a)

Kocamustafaogullari, G.: Pressure dependence of bubble departure diameter for water. Int. Commun. Heat Mass Transfer 10, 501-509 (1983b)

Kurihara, H., Myers, J.E.: The effects of superheat and surface roughness on boiling coefficients. AIChE J. 6, 83-91 (1960)

Li, Z.D., Zhang, L., Zhao, J.F., Li, H.X., Li, K., Wu, K.: Numerical simulation of bubble dynamics and heat transfer with transient thermal response of solid wall during pool boiling of FC-72. Int. J. Heat Mass Transfer 84, 409-418 (2015)

Mcfadden, P.W., Grassmann, P.: Relationships between bubble frequency and diameter during nucleate boiling. Int. J. Heat Mass Transfer 5, 169-172 (1962)

Mikic, B.B., Rohsenow, W.M., Griffith, P.: On bubble growth rates. Int. J. Heat Mass Transfer 13, 657-666 (1970)

Mukherjee, A., Kandlikar, S.G.: Numerical study of single bubbles with dynamic contact angle during nucleate pool boiling. Int. J. Heat Mass Transfer 50, 127-138 (2007)

Nikolayev, V., Garrabos, Y., Lecoutre, C., Charignon, T., Hitz, D., Chatain, D., Guillaument, R., Marre, S., Beysens, D.: Boiling crisis dynamics: Low gravity experiments at high pressure. Microgravity Sci. Technol. 27(4), 253-260 (2015)

Rohsenow, W.M.: A method of correlating heat transfer data for surface boiling of liquids. Trans. ASME 74, 969-976 (1952)

Rostamzadeh, A., Jafarpur, K., Rad, E.G.: Numerical investigation of pool nucleate boiling in nanofluid with lattice Boltzmann method. J. Theor. Appl. Mech. 54, 811-825 (2016)
Rothman, D.H., Keller, J.M.: Immiscible cellular-automaton fluids. J. Statistical Phys. 52, 1119-1127 (1980)

Ryu, S., Ko, S.: Direct numerical simulation of nucleate pool boiling using a two-dimensional lattice Boltzmann method. Nucl. Eng. Des. 248, 248-262 (2012)

Shan, X., Chen, H.: Lattice Boltzmann model for simulating flows with multiple phases and components. Phys. Rev. E 47, 1815 (1993)

Son, G., Ramanujapu, N., Dhir, V.K.: Numerical simulation of bubble merger process on a single nucleation site during pool nucleate boiling. J. Heat Transfer 124, 51-62 (2001)

Sun, T., Li, W.Z.: Three-dimensional numerical simulation of nucleate boiling bubble by lattice Boltzmann method. Comput. Fluids $\mathbf{8 8}$, 400-409 (2013a)

Sun, T., Li, W.Z., Yang, S.: Numerical simulation of bubble growth and departure during flow boiling period by lattice Boltzmann method. Int. J. Heat Fluid Flow 44, 120-129 (2013b)

Swift, M.R., Osborn, W.R., Yeomans, J.M.: Lattice Boltzmann simulation of nonideal fluids. Phys. Rev. Lett. 75, 830-833 (1995)

Siegel, R., Keshock, E.G.: Effects of reduced gravity on nucleate boiling bubble dynamics in saturated water. AIChE J. 10(4), 509-516 (1964)

Tien, C.L.: A hydrodynamic model for nucleate pool boiling. Int. J. Heat Mass Transfer 5, 533-540 (1962)

Wang, T.: VOSET Method Based on Adaptive Cartesian Grid with Application in Investigation on Bubble Dynamics in Electric Fields. Ph. D. Thesis. Xi' an Jiaotong University, China (2016)

Wallis, G.B.: One Dimensional Two-Phase Flow. McGarw-Hill, New York (1969)

Wu, K., Li, Z.D., Zhao, J.F., Li, H.X., Li, K.: Partial nucleate pool boiling at low heat flux: Preliminary ground test for SOBER-SJ10. Microgravity Sci. Technol. 28, 165-178 (2016)

Zhang, L., Li, Z.D., Li, K., Li, H.X., Zhao, J.F.: Influence of heater thermal capacity on bubble dynamics and heat transfer in nucleate pool boiling. Appl. Thermal Eng. 88, 118-126 (2015)

Zheng, H.W., Shu, C., Chew, Y.T.: A lattice Boltzmann model for multiphase flows with large density ratio. J. Comput. Phys. 218, 353-371 (2006)

Zuber, N.: Hydrodynamic aspect of boiling heat transfer. ph. d. thesis. University of California, Los Angeles (1959) 